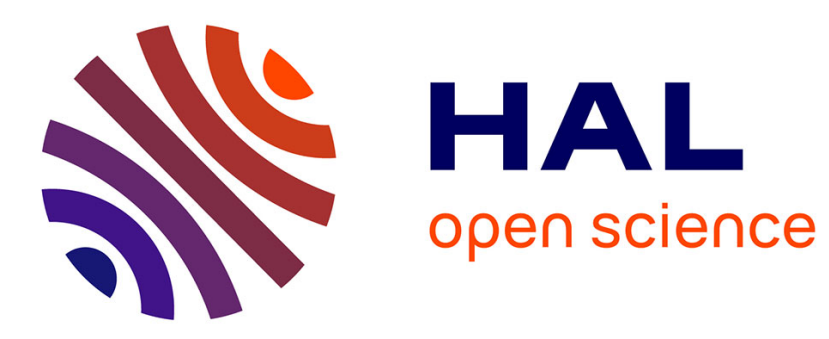

\title{
Mechanical and Imaging Models-based Image Registration
}

Kateřina Škardová, Matthias Rambausek, Radomir Chabiniok, Martin Genet

\section{To cite this version:}

Kateřina Škardová, Matthias Rambausek, Radomir Chabiniok, Martin Genet. Mechanical and Imaging Models-based Image Registration. VipIMAGE 2019 - VII ECCOMAS Thematic Conference on Computational Vision and Medical Image Processing, Oct 2019, Porto, Portugal. 10.1007/978-3-03032040-9_9. hal-02186828

\section{HAL Id: hal-02186828 https://hal.science/hal-02186828}

Submitted on 17 Jul 2019

HAL is a multi-disciplinary open access archive for the deposit and dissemination of scientific research documents, whether they are published or not. The documents may come from teaching and research institutions in France or abroad, or from public or private research centers.
L'archive ouverte pluridisciplinaire HAL, est destinée au dépôt et à la diffusion de documents scientifiques de niveau recherche, publiés ou non, émanant des établissements d'enseignement et de recherche français ou étrangers, des laboratoires publics ou privés. 


\title{
Mechanical and Imaging Models-based Image Registration
}

\author{
Kateřina Škardová, Matthias Rambausek, Radomír Chabiniok and Martin Genet
}

\begin{abstract}
Image registration plays an increasingly important role in many fields such as biomedical or mechanical engineering. Generally speaking, it consists in deforming a (moving) source image to match a (fixed) template image. Many approaches have been proposed over the years; if new model-free machine learning-based approaches are now beginning to provide robust and accurate results, extracting motion from images is still most commonly based on combining some statistical analysis of the images intensity and some model of the underlying deformation as initial guess or regularizer. These approaches may be efficient even for complex type of motion; however, any artifact in the source image (e.g., partial voluming, local decrease of signal-to-noise ratio or even local signal void), drastically deteriorates the registration. This paper introduces a novel approach of extracting motion from biomedical image series, based on a model of the imaging modality. It is, to a large extent,
\end{abstract}

Kateřina Škardová

Department of Mathematics, Faculty of Nuclear Sciences and Physical Engineering, Czech Technical University in Prague, Czech Republic

e-mail: katerina.solovska@fjfi.cvut.cz

Matthias Rambausek

Solid Mechanics Laboratory (LMS), École Polytechnique / CNRS / Paris Polytechnic Institute, France

e-mail: matthias.rambausek@polytechnique.edu

Radomír Chabiniok

Inria, Paris-Saclay University, France

Solid Mechanics Laboratory (LMS), École Polytechnique / CNRS / Paris Polytechnic Institute, France

School of Biomedical Engineering \& Imaging Sciences (BMEIS), St Thomas' Hospital, King's College London, United Kingdom

e-mail: radomir.chabiniok@inria.fr

Martin Genet

Solid Mechanics Laboratory (LMS), École Polytechnique / CNRS / Paris Polytechnic Institute, France; Paris-Saclay University, France

e-mail: martin.genet@polytechnique.edu 
independent of the type of model and image data-the pre-requisite is to incorporate biomechanical constraints into the motion of the object (organ) of interest and being able to generate data corresponding to the real image, i.e., having an imaging model at hand. We will illustrate the method with examples of synthetically generated 2D tagged magnetic resonance images.

\section{Introduction}

Image registration plays an increasingly important role in many fields such as biomedical (Wang and Amini, 2012; Tobon-Gomez et al., 2013) or mechanical (Bornert et al., 2012; Sutton and Hild, 2015) engineering. Taking cardiology as an example, the heart represents a particular organ in the field of medical imaging since $(i)$ a large number of modalities and image types have been developed and the process of combining them -in which registration represents a crucial step- may maximize the amount of information for diagnostic purposes with possible outcomes in long-term management of patients' therapy (Helsen et al., 2018; Rutz et al., 2017); and (ii) the heart is a moving organ and registering a motion series of images allows to extract its functional characteristics (Wang and Amini, 2012; Tobon-Gomez et al., 2013). While ( $i$ ) is expected to play an increasing role in the near future when a comprehensive information across medical fields will be sought for, (ii) is already a very active topic nowadays, aiming at an accurate and reproducible description of heart function. It is connecting the communities of imaging, image processing, biomedical and mechanical engineering and statistical methods. Image-based motion data are paramount, for instance, in designing personalized biomechanical cardiac models (Smith et al., 2011; Krishnamurthy et al., 2013; Finsberg et al., 2018), which could be used for augmented diagnosis (Chabiniok et al., 2012; Xi et al., 2016) and treatments (Sermesant et al., 2012; Rausch et al., 2017).

Generally speaking, image registration consists in deforming a (moving) source image to match a (fixed) template image. Although new model-free machine learningbased approaches are now beginning to provide robust and accurate results (Suinesiaputra et al., 2015; Qin et al., 2018), extracting motion from images is still most commonly based on combining some statistical analysis of the images intensity and some model of the underlying deformation as initial guess or regularizer (Shi et al., 2012; Bornert et al., 2012; Tobon-Gomez et al., 2013). These approaches may be efficient even for complex type of motion; however, any artifact in the source image (e.g., partial voluming, local decrease of signal-to-noise ratio or even local signal void), drastically deteriorates the registration. Mathematical, geometrical or mechanical regularization can help to alleviate the issue (Christensen, Rabbitt, and Miller, 1996; Veress, Gullberg, and Weiss, 2005; Genet, C. T. Stoeck, et al., 2016). However, it is intrinsic to the intensity-based approach, and problems remain. For instance, radial strains are systematically underestimated in 3D tagging cardiac images (Tobon-Gomez et al., 2013; Genet, C. Stoeck, et al., 2018). 
This paper introduces a novel approach of extracting motion from biomedical image series, based on a model of the imaging modality. It is, to a large extent, independent of the type of image -the only pre-requisite is to be able to generate a realistic image associated to a given shape of the considered object, i.e., having an imaging model at hand. We will illustrate the method with examples of synthetically generated tagged magnetic resonance images.

\section{Methods}

\subsection{Problem setting}

$\tilde{I}_{0} \& \tilde{I}$ are two images representing the same body $\mathcal{B}$ at two instants $t_{0} \& t$ :

$$
\tilde{I}_{0}:\left\{\begin{array}{l}
\square_{0} \rightarrow \mathbb{R} \\
\underline{X} \mapsto \tilde{I}_{0}(\underline{X})
\end{array} \quad, \quad \tilde{I}:\left\{\begin{array}{l}
\square \rightarrow \mathbb{R} \\
\underline{x} \mapsto \tilde{I}(\underline{x})
\end{array},\right.\right.
$$

where $\square_{0} \& \square$ are the image domains at $t_{0} \& t$, which are usually identical. The domains occupied by the body $\mathcal{B}$ at $t_{0} \& t$ are denoted $\Omega_{0} \& \Omega$, respectively. The problem is to find the smooth mapping $\underline{\Phi}$ between materials points of the reference and deformed domains:

$$
\underline{\Phi}:\left\{\begin{array}{l}
\Omega_{0} \rightarrow \Omega \\
\underline{X} \mapsto \underline{x}=\underline{\Phi}(\underline{X})
\end{array},\right.
$$

where $\underline{X} \& \underline{x}$ denote the position of a given material point in the reference and deformed configurations. Equivalently, the problem can be formulated in terms of the smooth displacement field $\underline{U}$ :

$$
\underline{U}:\left\{\begin{array}{l}
\Omega_{0} \rightarrow \mathbb{R}^{3} \\
\underline{X} \mapsto \underline{U}(\underline{X})=\underline{\Phi}(\underline{X})-\underline{X}
\end{array} .\right.
$$

Because of intrinsic ill-posedness, it is formulated as a minimization problem:

$$
\text { find } \underline{\Phi^{\text {sol }}}=\operatorname{argmin}_{\{\underline{\Phi}\}}\left\{\Psi^{\text {cor }}(\underline{\Phi})\right\},
$$

where $\Psi^{\text {cor }}$ is the "correlation energy", or image similarity metric, which is in general not quadratic, but which we assume convex, at least in the neighborhood of the solution. Many approaches have been proposed to regularize this ill-posed problem (Christensen, Rabbitt, and Miller, 1996; Veress, Gullberg, and Weiss, 2005; Mansi et al., 2011; Wang and Amini, 2012; Tobon-Gomez et al., 2013), which will not be discussed in details here. Specifically, details on the equilibrium gap regularization, an efficient mechanistic approach, can be found in (Claire, Hild, and Roux, 2004; Genet, C. Stoeck, et al., 2018; Lee and Genet, 2019). 


\subsection{Intensity-based approaches}

In intensity-based approaches, the following correlation energy is generally used:

$$
\Psi^{\mathrm{cor}}(\underline{\Phi})=\frac{1}{2} \int_{\Omega_{0}}\left(\tilde{I}(\underline{\Phi}(\underline{X}))-\tilde{I}_{0}(\underline{X})\right)^{2} d \Omega_{0}
$$

The main drawback of this method is that it consists in mapping both images directly, so that any artifact in the images, especially partial voluming, will pollute the correlation. Here we propose to circumvent this problem by using, besides the geometrical model of the object to track, a model of the imaging process. Thus we can generate synthetic images from the model at every time step, and look for the mapping that best matches the synthetic and acquired images.

\subsection{Proposed approach}

\subsubsection{Imaging model}

For a body $\mathcal{B}$ occupying the spatial domain $\Omega_{0}$, we define the "pure" cine-like image:

$$
I_{\Omega_{0}}^{\text {cine }}(\underline{X}):=\mathbb{1}_{\Omega_{0}}(\underline{X})=\left\{\begin{array}{l}
1 \text { if } \underline{X} \in \Omega_{0} \quad \forall \underline{X} \in \square_{0}, \\
0 \text { otherwise }
\end{array}\right.
$$

as well as a "pure" tagging-like image:

$$
I_{\Omega_{0}}^{\mathrm{tag}}(\underline{X}):=\mathbb{1}_{\Omega_{0}}(\underline{X})\left|\sin \left(\underline{k} \cdot\left(\underline{X}-\underline{X_{0}}\right)\right)\right| \quad \forall \underline{X} \in \square_{0},
$$

where $\underline{k}$ and $X_{0}$ are tagging pattern parameters. Tagged images are usually combined to form a grid image, so the associated model (taking $\underline{X_{0}}=0$ ) is:

$$
I_{\Omega_{0}}^{\mathrm{grid}}(\underline{X}):=\mathbb{1}_{\Omega_{0}}(\underline{X}) \sqrt[n]{\prod_{i=1}^{n}\left|\sin \left(\pi X_{i} / s\right)\right|} \quad \forall \underline{X} \in \square_{0},
$$

where $s$ is the tag line distance and $n$ the image dimension.

Once the body has moved and occupies the domain $\Omega=\Phi\left(\Omega_{0}\right)$, the deformed image is simply:

$$
I_{\Omega_{0}, \Phi}(\underline{x})=I_{\Omega_{0}}\left(\underline{\Phi}^{-1}(\underline{x})\right) \quad \forall \underline{x} \in \square .
$$

Remark 1 For cine-like images, since they do not require to follow material points but only domain boundaries, we can express the deformed image solely from the deformed domain:

$$
I_{\Omega_{0}, \Phi}^{\text {cine }}(\underline{x})=\mathbb{1}_{\Omega_{0}}\left(\underline{\Phi}^{-1}(\underline{x})\right)=\mathbb{1}_{\Omega}(\underline{x})=I_{\Omega}^{\text {cine }}(\underline{x}) \quad \forall \underline{x} \in \square .
$$


However, it is not possible for tagging-like images, as the mapping is required to track material points.

Because MR has limited bandwidth, the frequency content of actual images is only sampled within a box window, which means, in space, that they are convoluted by a cardinal sine kernel:

$$
\tilde{I}_{\Omega_{0}, \Phi}(\underline{x})=\int_{\square} I_{\Omega_{0}, \Phi}(\underline{y}) v(\underline{y}-\underline{x}) d \square \quad \forall \underline{x} \in \square,
$$

with:

$$
v(\underline{z}):=\prod_{i} \operatorname{sinc}\left(\frac{z_{i}}{\Delta_{i}}\right),
$$

where $\underline{\Delta}$ denotes the image resolution.

\subsubsection{Correlation energy}

We now seek the mapping that best matches the generated and actual images, which minimizes the following correlation energy:

$$
\Psi^{\mathrm{cor}}(\underline{\Phi})=\frac{1}{2} \int_{\Omega_{0}}\left(\tilde{I}_{\Omega_{0}, \Phi}(\underline{\Phi}(\underline{X}))-\tilde{I}(\underline{\Phi}(\underline{X}))\right)^{2} d \Omega_{0},
$$

where:

$$
\tilde{I}_{\Omega_{0}, \Phi}(\underline{\Phi}(\underline{X}))=\int_{\square} I_{\Omega_{0}, \Phi}(\underline{y}) v(\underline{y}-\underline{\Phi}(\underline{X})) d \square \quad \forall \underline{X} \in \Omega_{0} .
$$

Remark 2 In case the effect of image resolution is neglected, i.e., the convolution kernel is replaced by Dirac function, this leads to:

$$
\tilde{I}_{\Omega_{0}, \Phi}(\underline{\Phi}(\underline{X}))=I_{\Omega_{0}, \Phi}(\underline{\Phi}(\underline{X}))=I_{\Omega_{0}}(\underline{X})
$$

\section{Results}

Four sets of synthetic images were generated in order to test the proposed method, representing both rigid (translation \& rotation) and non-rigid (compression \& shear) transformations of a simple square object. All sequences were 2D, 100x100 pixels, 30 frames long. Tagging magnetic resonance image model (8) was used, with $\mathrm{s}=10$ pixels. For all transformations, a noise-free image was generated (i.e., $\mathrm{SNR}=+\infty$ ), as well as noisy images, by adding zero-mean gaussian noise with various standard deviations: 0.1 (i.e., $\mathrm{SNR}=10), 0.2(\mathrm{SNR}=5) \& 0.3(\mathrm{SNR}=3)$. In a first step, well resolved images were used, such that the effect of image resolution and discretization can be neglected, and expression (15) can be used. 
All sets of images were registered. Details on the solution procedure can be found in (Genet, C. Stoeck, et al., 2018), and the code can be found at https://gitlab. inria.fr/mgenet/dolfin_dic. Figures 1, 2, 3 \& 4 show the registration results, in the form of the warped mesh on top of the synthetic images (with various levels of noise). The quality of the registration is clearly seen, except for the rotation case (Figure 2) where one node ends up outside the image. Note that this could be alleviated by using some proper mechanical regularization in order to filter out the non physical deformation patterns (Genet, C. Stoeck, et al., 2018; Berberoglu et al., 2019). In other cases, even for quite noisy images (as illustrated in Figure 3), the registration is almost perfect.

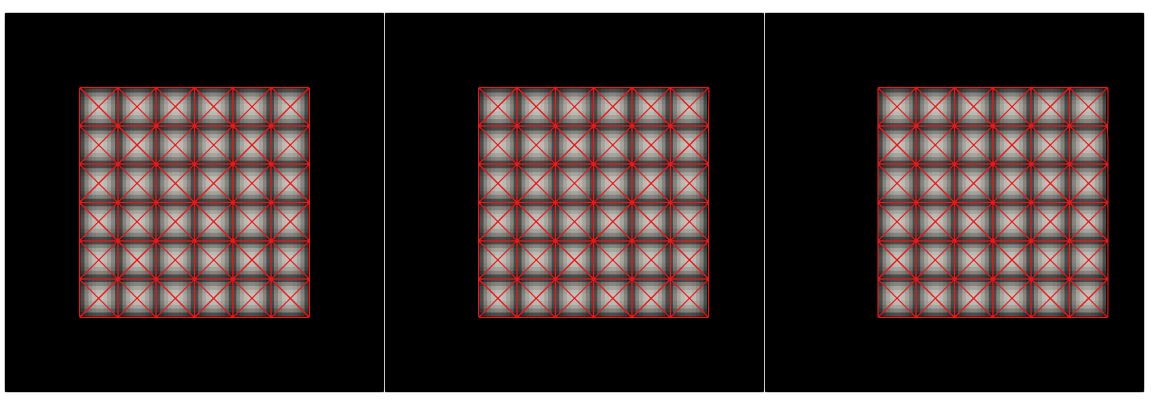

Fig. 1 Registration results on the translation case, for the noise-free images.

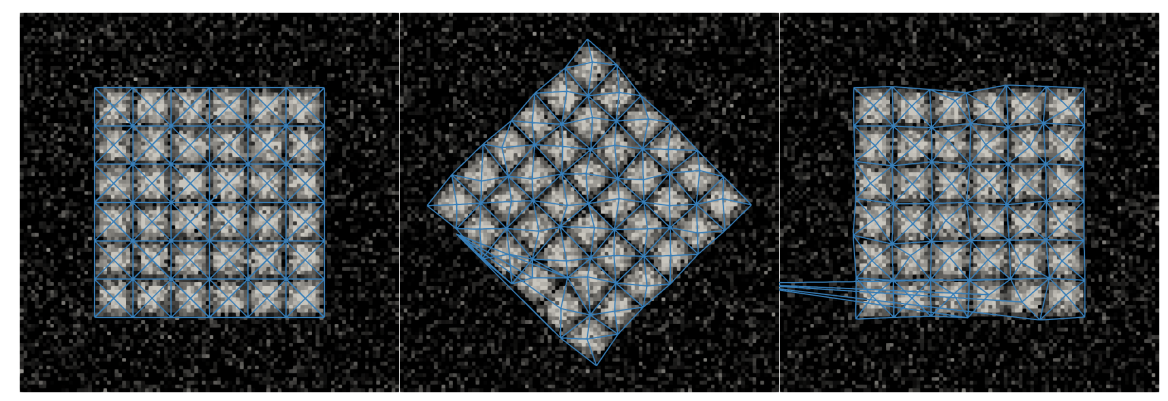

Fig. 2 Registration results on the rotation case, for the $\mathrm{SNR}=5$ images. The registration fails at one node, which could be prevented by using proper mechanical regularization.

This is better quantified in Figure 5, which shows, for all tested transformations and SNR, the normalized root mean square displacement error: 


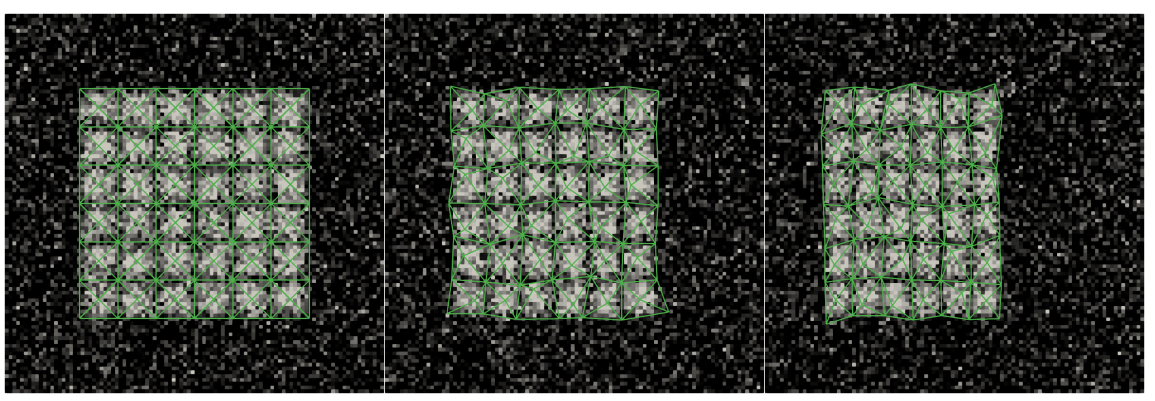

Fig. 3 Registration results on the compression case, for the $\mathrm{SNR}=3$ images.

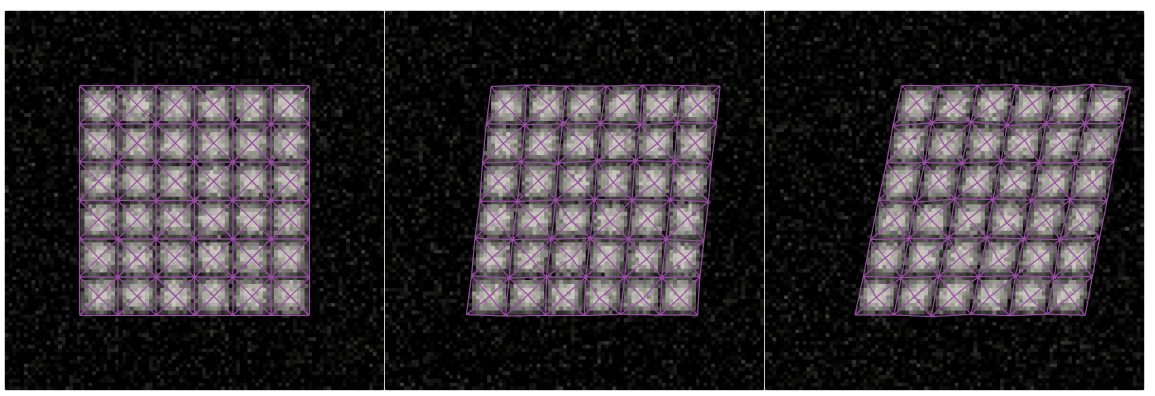

Fig. 4 Registration results on the shear case, for the SNR $=10$ images.

$$
R M S E_{U}=\frac{\sqrt{\frac{1}{T} \int_{0}^{T} \frac{1}{\left|\Omega_{0}\right|} \int_{\Omega_{0}}\left\|\underline{U}-\underline{U^{\mathrm{ref}}}\right\|^{2}}}{\sqrt{\frac{1}{T} \int_{0}^{T} \frac{1}{\left|\Omega_{0}\right|} \int_{\Omega_{0}}\left\|\underline{U^{\mathrm{ref}}}\right\|^{2}}},
$$

where $\underline{U^{\text {ref }}}$ is the exact solution used to generate the synthetic images. A similar trend is found in Figure 6, which shows the impact of SNR on the normalized root mean square image similarity error:

$$
R M S E_{I}=\frac{\sqrt{\frac{1}{T} \int_{0}^{T} \frac{1}{\left|\Omega_{0}\right|} \int_{\Omega_{0}}\left(\tilde{I}_{\Omega_{0}, \Phi}(\underline{\Phi}(\underline{X}))-\tilde{I}(\underline{\Phi}(\underline{X}))\right)^{2}}}{\sqrt{\frac{1}{T} \int_{0}^{T} \frac{1}{\left|\Omega_{0}\right|} \int_{\Omega_{0}}(\tilde{I}(\underline{\Phi}(\underline{X})))^{2}}} .
$$




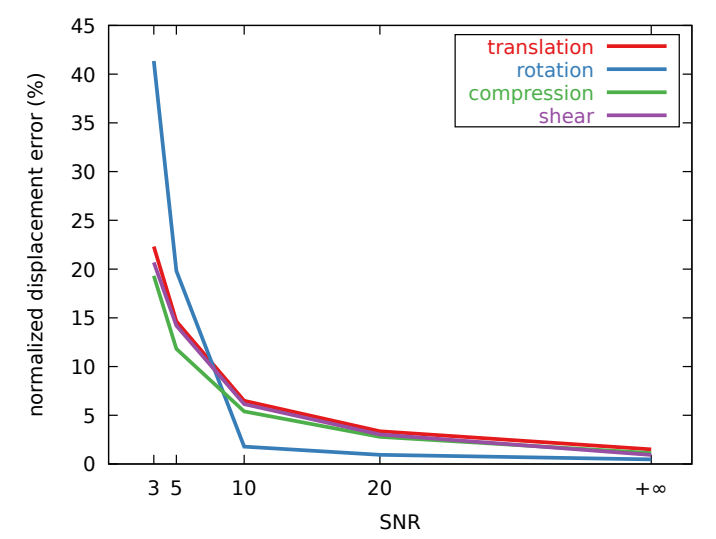

Fig. 5 Impact of SNR on the registration, in terms of normalized root mean square displacement error.

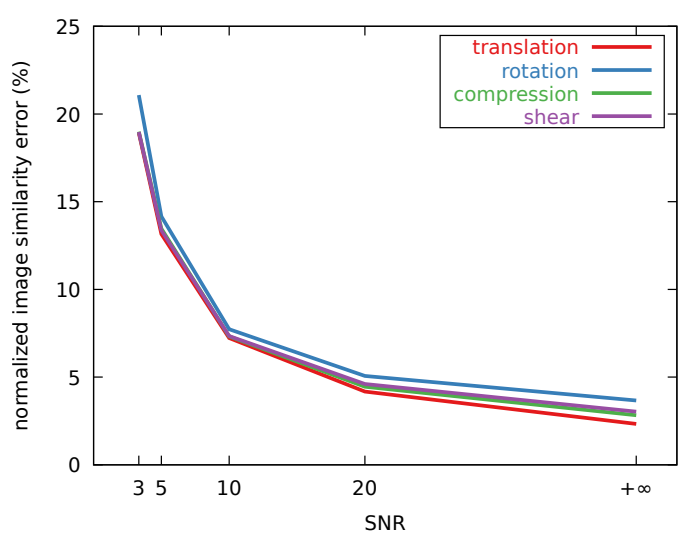

Fig. 6 Impact of SNR on the registration, in terms of normalized root mean square image similarity error.

\section{Conclusion \& Perspectives}

A novel image registration method has been introduced, which uses a model of the imaging device itself. Formulated and solved using the finite element method, it can naturally be used in conjunction with existing mechanics-based regularization approaches. It was tested on simple rigid and non-rigid transformations, and was shown to perform well on all of them.

The set of testing sequences will be extended in the future, notably to include the impact of image resolution. More complicated shapes and transformation will be tested as well. In fine, the method will be tested on real images, and could alleviate the image resolution-induced underestimation of radial strain classically found in $3 \mathrm{D}$ tagged magnetic resonance images. 


\section{Acknowledgements}

The work was supported by the Inria-UT Southwestern Medical Center Dallas Associated team ToFMOD, and partially by the Ministry of Health of the Czech Republic (project No. NV19-08-00071).

\section{References}

Berberoglu, E. et al. (2019). "Validation of Finite Element Image Registration-Based Cardiac Strain Estimation from Magnetic Resonance Images". In: 90th Annual Meeting of the International Association of Applied Mathematics and Mechanics (GAMM).

Bornert, M. et al. (2012). "Digital Image Correlation". In: Full-Field Measurements and Identification in Solid Mechanics. Ed. by M. Grédiac, F. Hild, and A. Pineau. John Wiley \& Sons, Inc. DoI: 10.1002/9781118578469 . ch6.

Chabiniok, R. et al. (2012). "Estimation of Tissue Contractility from Cardiac CineMRI Using a Biomechanical Heart Model". In: Biomechanics and Modeling in Mechanobiology 11.5. Dor: 10.1007/s10237-011-0337-8.

Christensen, G. E., R. D. Rabbitt, and M. I. Miller (1996). "Deformable Templates Using Large Deformation Kinematics". In: IEEE transactions on image processing : a publication of the IEEE Signal Processing Society 5.10. DoI: 10.1109/83.536892.

Claire, D., F. Hild, and S. Roux (2004). “A Finite Element Formulation to Identify Damage Fields: The Equilibrium Gap Method". In: International Journal for Numerical Methods in Engineering 61.2. DoI: 10.1002/nme . 1057.

Finsberg, H. et al. (2018). "Efficient Estimation of Personalized Biventricular Mechanical Function Employing Gradient-Based Optimization”. In: International Journal for Numerical Methods in Biomedical Engineering 34.7. DoI: 10.1002/ cnm. 2982.

Genet, M., C. Stoeck, et al. (2018). "Equilibrated Warping: Finite Element Image Registration with Finite Strain Equilibrium Gap Regularization". In: Medical Image Analysis. Dor: 10.1016/j .media.2018.07.007.

Genet, M., C. T. Stoeck, et al. (2016). "Finite Element Digital Image Correlation for Cardiac Strain Analysis from 3D Whole-Heart Tagging”. In: 24th Annual Meeting of the International Society for Magnetic Resonance in Medicine (ISMRM2016).

Helsen, F. et al. (2018). “Advanced Imaging to Phenotype Patients With a Systemic Right Ventricle”. In: Journal of the American Heart Association 7.20. DoI: 10. 1161/JAHA. 118.009185.

Krishnamurthy, A. et al. (2013). "Patient-Specific Models of Cardiac Biomechanics". In: Journal of computational physics 244. Dor: 10 . 1016/ j . j cp . 2012 . 09.015.

Lee, L. C. and M. Genet (2019). "Validation of Equilibrated Warping-Image Registration with Mechanical Regularization-On 3D Ultrasound Images". In: Functional Imaging and Modeling of the Heart (FIMH). Ed. by Y. Coudière et al. 
Vol. 11504. Cham: Springer International Publishing. DoI: 10 . 1007/978-3Q30-21949-9_36.

Mansi, T. et al. (2011). "iLogDemons: A Demons-Based Registration Algorithm for Tracking Incompressible Elastic Biological Tissues". In: International Journal of Computer Vision 92.1. DoI: 10.1007/s11263-010-0405-z.

Qin, C. et al. (2018). "Joint Learning of Motion Estimation and Segmentation for Cardiac MR Image Sequences". In: International Conference on Medical Image Computing and Computer-Assisted Intervention (MICCAI).

Rausch, M. K. et al. (2017). "A Virtual Sizing Tool for Mitral Valve Annuloplasty”. In: International Journal for Numerical Methods in Biomedical Engineering 33.2. DOI: $10.1002 / \mathrm{cnm} .2788$.

Rutz, T. et al. (2017). "Evolution of Right Ventricular Size over Time after Tetralogy of Fallot Repair: A Longitudinal Cardiac Magnetic Resonance Study". In: European Heart Journal - Cardiovascular Imaging 18.3. Dor: 10 . 1093/ehjci/ jew273.

Sermesant, M. et al. (2012). "Patient-Specific Electromechanical Models of the Heart for the Prediction of Pacing Acute Effects in CRT: A Preliminary Clinical Validation." In: Medical Image Analysis 16.1. Dor: 10.1016/j . media. 2011. 07.003.

Shi, W. et al. (2012). "A Comprehensive Cardiac Motion Estimation Framework Using Both Untagged and 3-D Tagged MR Images Based on Nonrigid Registration". In: IEEE Transactions on Medical Imaging 31.6. DOI: 10.1109/TMI . 2012.2188104.

Smith, N. P. et al. (2011). "euHeart: Personalized and Integrated Cardiac Care Using Patient-Specific Cardiovascular Modelling”. In: Interface focus 1.3. Dor: $10.1098 / \mathrm{rsfs} .2010 .0048$.

Suinesiaputra, A. et al. (2015). "Big Heart Data: Advancing Health Informatics Through Data Sharing in Cardiovascular Imaging". In: IEEE Journal of Biomedical and Health Informatics 19.4. DoI: 10.1109/JBHI.2014.2370952.

Sutton, M. A. and F. Hild (2015). "Recent Advances and Perspectives in Digital Image Correlation". In: Experimental Mechanics 55.1. Dor: 10 . 1007/s11340015-9991-6.

Tobon-Gomez, C. et al. (2013). "Benchmarking Framework for Myocardial Tracking and Deformation Algorithms: An Open Access Database". In: Medical Image Analysis 17.6. Dor: 10.1016/j .media.2013.03.008.

Veress, A. I., G. T. Gullberg, and J. A. Weiss (2005). "Measurement of Strain in the Left Ventricle during Diastole with Cine-MRI and Deformable Image Registration". In: Journal of Biomechanical Engineering 127.7. Dor: 10.1115/ 1.2073677.

Wang, H. and A. A. Amini (2012). "Cardiac Motion and Deformation Recovery from MRI: A Review". In: IEEE transactions on medical imaging 31.2. DoI: 10.1109/TMI.2011.2171706.

Xi, C. et al. (2016). "Patient-Specific Computational Analysis of Ventricular Mechanics in Pulmonary Arterial Hypertension". In: Journal of Biomechanical Engineering 138.11. DoI: 10.1115/1.4034559. 